\title{
Strain-induced Differentiation of Mesenchymal Stem Cells
}

\author{
Khalil Moussi, Member, IEEE, Dina B. Abusamra, Omar Yassine, \\ Jasmeen Merzaban, Jürgen Kosel, Senior Member, IEEE
}

\begin{abstract}
Directing the fate of human mesenchymal stem/stromal cells (hMSCs) toward bone formation using mechanical strain is a promising approach in regenerative medicine related to bone diseases. Numerous studies have evaluated the effects of vibration or cyclic tensile strain on MSCs towards developing a mechanically-based method for stimulating differentiation. Here, we study the differentiation of hMSCs cultured on elastic polydimethylsiloxane (PDMS) membrane, which is magnetically actuated to induce periodically varying strain. The strain distribution across the membrane was calculated by finite-element modeling and demonstrates three main areas of different strain amplitudes. The strain effect on the hMSCs was evaluated by measuring the mineralization of differentiated hMSCs using Alizarin $S$ red stain. The results indicate a straindependent differentiation of hMSCs, where the highest region of strain on the membrane resulted in the most accelerated differentiation. Osteogenic differentiation was achieved as early as two weeks, which is significantly sooner than control hMSCs treated with osteogenic media alone.
\end{abstract}

Keywords-Mesenchymal stem cells, Mechanical strain, Osteogenic differentiation.

\section{INTRODUCTION}

$\mathbf{H}$ Uman mesenchymal stem/stromal cells (hMSCs) continue to be a cornerstone in the fields of basic science and regenerative medicine, mainly due to their capacity to propagate in culture, while retaining their differentiation potential towards several lineages [1]-[4]. Regardless of the tissue of origin, all hMSCs can differentiate in vitro into osteoblasts, chondrocytes, and adipocytes among other cell types [2], [5]. In addition, hMSCs possess immunomodulatory properties [3], [4], [6]. For these reasons and others, hMSCs are great prospects in therapy and tissue engineering. Developing means of understanding and controlling parameters that affect hMSC differentiation toward specific lineages could significantly benefit modern regenerative medicine [1][3], [7]. The interaction between MSCs and their niches to regulate tissue regeneration is a complex matter and is still not fully understood. Osteogenic differentiation is influenced by molecular factors, such as cytokines, hormones and transcription factors, present within their environment [2], [8][10]. Furthermore, several studies indicate the substantial role

K. Moussi, O. Yassine, and J. Kosel are with the Computer, Electrical and Mathematical Science and Engineering division at King Abdullah University of Science and Technology, Thuwal, Saudi Arabia.

D.B. Abusamra and J. Merzaban are with the Biological Environmenta Science and Engineering division at King Abdullah University of Science and Technology, Thuwal, Saudi Arabia.

E-mail:\{khalil.moussi, jasmeen.merzaban, jurgen.kosel\}@ kaust.edu.sa. of exercise and mechanical factors in directing the differentiation of MSCs. For instance, exercise elicits a significantly higher potential towards osteogenic (with a concomitant inhibition towards adipogenic) differentiation of MSCs in both mouse and human subjects, resulting in increased bone mass compared to sedentary controls [11]-[13]. This is thought to indicate that cytoskeletal tension, along with mechanical and biochemical cues, help direct differentiation mainly through controlling the cell shape, which is a key regulator of stem cell fate [14]-[16]. For example, Ruiz et al. [17] applied microscale-patterning approaches, to control the geometries of both two-dimensional (2D) and three-dimensional (3D) multicellular structures of hMSCs. These authors reported that in the presence of soluble factors, permitting both osteogenic and adipogenic differentiation, the high strain region at the edges of the multicellular structures selectively differentiated into the osteogenic lineage, whereas those of the low strain region became adipocytes. Moreover, McBeath et al. [18] used a microcontact printing technique on a flat surface of polydimethylsiloxane (PDMS) to demonstrate the effect of cell shape in controlling stem cell differentiation. Similarly, stem cells growing on nanostructured substrates showed different shape, osteogenic lineage commitment, and cytoskeleton remodeling [19]-[24]. There is still much to be revealed about the contribution of the mechano-transduction to this mechanism and some studies suggest that the cytoskeleton, and specifically microtubules, play a key role in controlling the mechanical stability inside of the cell to the centrosome [25].

Different approaches have been applied to improve selective osteo-induction and, consequently, to reduce the time required to achieve differentiation. Stimulation with pulsed electromagnetic field vibration is clinically used to accelerate and finalize the healing process of a fresh fracture, or a fracture at risk of nonunion, and to enhance the spontaneous repair capability of delayed union, pseudoarthrosis or osteoporosis conditions. A recent study demonstrated that lowfrequency electromagnetic fields enhance the chondrogenic differentiation of hMSCs, while higher frequencies induce osteoblast differentiation [26]. Several studies have also implicated the role of mechanical stimuli, such as compression, fluid shear strain and magnetic stimulation using magnetic particles along with ultrasonic waves, in triggering the differentiation of hMSCs towards osteocytes [1], [11], [27]-[41]. However, the main drawback of the majority of these studies 
(a)

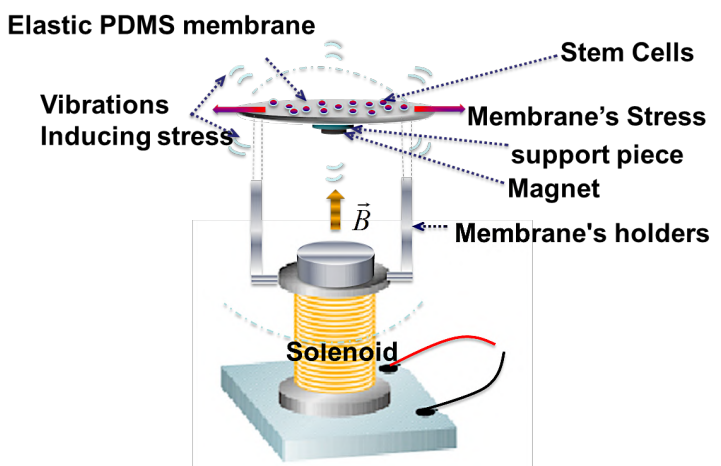

(b)

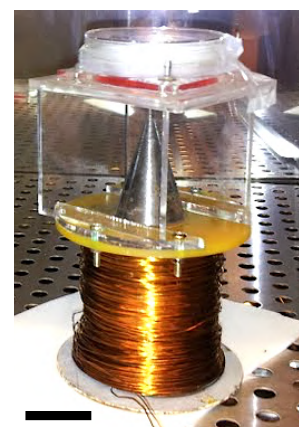

(c)

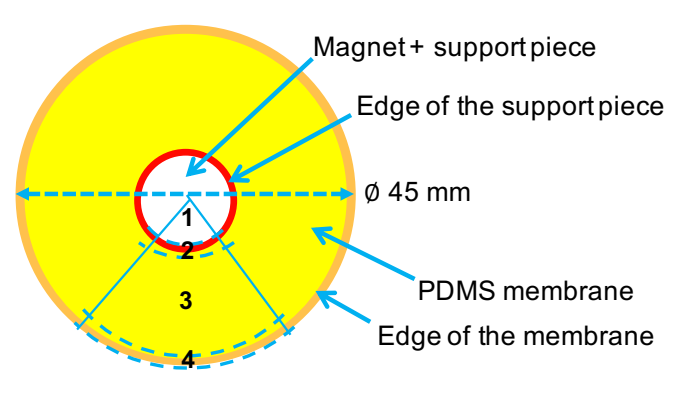

Fig. 1: Experimental setup. (a) Schematic of the experimental setup including an elastic PDMS membrane onto which cells are seeded, a permanent magnet in the center of the membrane and an electromagnet to drive the vibration. (b) Photograph of the setup (scale bar is $25 \mathrm{~mm}$ ). (c) Schematic showing the elastic membrane and its different zones that were used for evaluating the stem cell differentiation.

is that they used molecular products or cues (i.e., growth factors) to inhibit or exhibit specific outcomes. Understanding the actual force needed to accelerate the differentiation of hMSC toward different lineages (i.e., osteoblasts for instance) is less understood. Thus, in this study, we investigate the effect of external mechanical forces without the addition of any external cues. hMSCs were maintained on an elastic membrane that was periodically stretched generating a measurable force on the membrane. Subsequently, a strain was provided by the vibration of this membrane generated by the interaction of a permanent magnet at the membrane's center with an electromagnet. This unique setup allowed us to determine the effect of the strain distribution over the membrane on the differentiation of hMSCs.

\section{MATERIALS AND METHODS}

\section{A. Setup}

The experimental setup (Fig. 1(a-b)) is composed of a solenoid with a membrane holder fixed on top of it. The holder is made of polymethyl methacrylate (PMMA) and is composed of three parts fabricated by laser cutting. The membrane was sandwiched between two identical PMMA pieces having a circular hole with a diameter $10 \mathrm{~mm}$ smaller than the membrane to fix its edge. As the cells need to be dispersed in a liquid medium, a PMMA ring with a thickness of $1 \mathrm{~mm}$ was added on top of this membrane holder to form a container. This assembly was mounted on top of two vertical PMMA plates, which were fixed to the solenoid. The distance between solenoid and membrane was around $1 \mathrm{~cm}$. Finally, the setup was placed inside of an incubator, and the solenoid was connected to an $\mathrm{AC}$ amplifier (NF, HSA 4012) in combination with a function generator (Agilent 33250A) and the membrane vibrated at a frequency of $24 \mathrm{~Hz}$. The elastic membrane (Fig. 1(c)) was made from polydimethylsiloxane (PDMS), which is biocompatible and has convenient elastic properties [42], [43]. Briefly, liquid PDMS was mixed and spun on top of a glass slide. The thickness of the PDMS membrane $(100 \mu \mathrm{m})$ was controlled by adjusting the spin coating parameters. Then, the PDMS was cured at $75^{\circ} \mathrm{C}$. Later, the PDMS was peeled off and cut into the desired circular shape with a diameter of $45 \mathrm{~mm}$. A support piece made of PMMA with diameter and thickness of $10 \mathrm{~mm}$ and $1 \mathrm{~mm}$, respectively, was fixed to the center of the membrane at its bottom side and a permanent ferrite magnet of diameter and thickness of $5 \mathrm{~mm}$ and $1 \mathrm{~mm}$, respectively, was attached to this supporting piece.

\section{B. Cells and reagents}

Human bone marrow derived mesenchymal stem/stromal cells (hMSCs) were purchased from AllCells. All the experiments were performed using MSCs in their 3-5th passage. An Alizarin red S kit for measuring calcium deposits staining in osteocytes was purchased from IHC World. Human plasma Fibronectin was purchased from Gibco, Invitrogen. MesenCult TM proliferation kit (human) and MesenCult ${ }^{\mathrm{TM}}$ Osteogenic differentiation kit (human) were obtained from Stem Cell Technologies.

\section{Cell culture setup}

In order to utilize the membrane as a cell growth substrate, it was first sterilized using 70\% ethanol and exposed to UVlight for 3 hours, washed with phosphate buffer saline (PBS) and then coated with $10 \mu \mathrm{g} / \mathrm{ml}$ fibronectin for 4 hours at room temperature. A total of $1 \times 10^{6}$ cells were inoculated onto the membrane and cultured using MesenCult medium supplemented with $10 \%$ MesenCult $^{\mathrm{TM}}$ Stem Cell Stimulatory Supplements and $2 \mathrm{mM}$ L-glutamine. Then, the membrane with hMSCs was placed into a humidified cell culture incubator $\left(37^{\circ} \mathrm{C}, 5 \% \mathrm{CO}_{2}\right)$. After 24 hours, MSCs reached $80-90 \%$ confluency and securely lodged onto the membrane of the electromagnetic vibration system. To qualitatively determine the differentiation efficiency of cultured cells an equal number of cells were seeded into fibronectin coated $60 \mathrm{~mm}$ tissue culture dish with the same medium but without vibration (negative control) or with osteogenic differentiation medium containing 15\% of Osteogenic Stimulatory Supplements, 3.5 
(a)

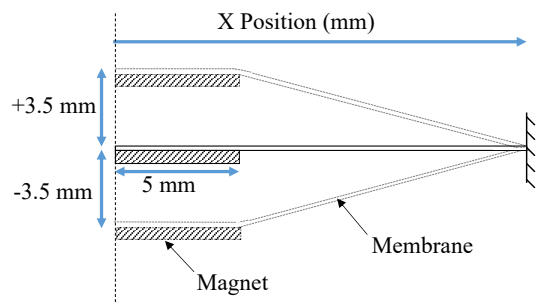

(b)

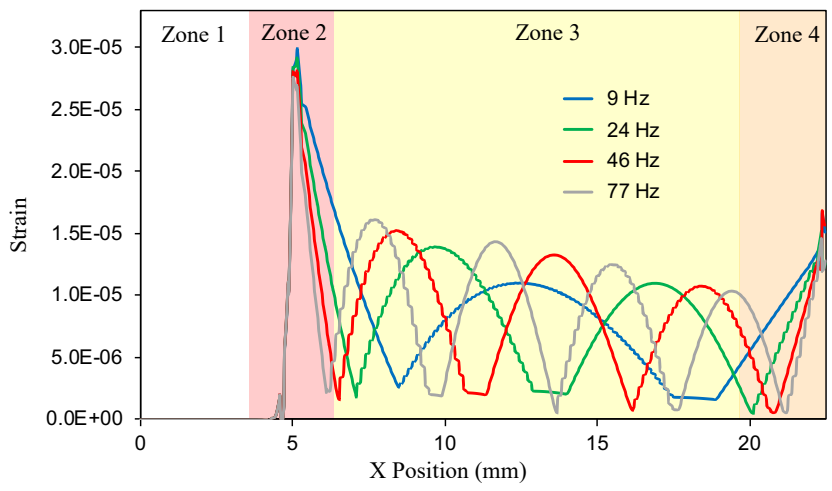

Fig. 2: (a) 2D schematic of the membrane oscillation. (b) Strain distribution with respect to the Eigen-frequencies at steady state $(\mathrm{z}=0 \mathrm{~mm})$.

$\mathrm{mM} \beta$-Glycerophosphate, $0.1 \mathrm{nM}$ Dexamethasone and 50 $\mu \mathrm{g} / \mathrm{mL}$ Ascorbic Acid (positive control). The control groups of cells were incubated in the same incubator under the same experimental conditions but with no exposure to the electromagnetic fields. Fresh medium was added every day to reach $10 \mathrm{ml}$ in total to maintain constant fluid perturbation (and thus fluid shear strain) regardless of evaporation.

\section{Analysis of differentiation of hMSCs}

After 14,17 and 21 days, hMSCs cultured on the membrane as well as controls were rinsed twice with PBS, fixed with $4 \%$ paraformaldehyde at room temperature for 20 min and washed with distilled water. To detect osteogenic induction, the membrane was cut in quarters, stained with Alizarin red solution for $5 \mathrm{~min}$ and then dehydrated using an alcohol gradient. Each membrane quarter was analyzed by taking images from the center to the edge using 10x magnitude lens (Nikon Eclipse TS-100), which were studied using ImageJ software. The average intensity of each region was calculated from the red channel split image.

\section{RESULTS AND DISCUSSIONS}

Under electromagnetic excitation, the elastic membrane undergoes harmonic oscillation induced by force acting between the permanent magnet and the electromagnet. A frequency analysis was performed using finite element modeling (FEM) (COMSOL Multiphysics software) to find the resonant frequencies of the vibrating system consisting of the membrane, support piece, and magnet. To simplify the simulation a 2D axisymmetric analysis was performed (exploiting circular symmetry), as shown in Fig. 2(a). The boundary (a)

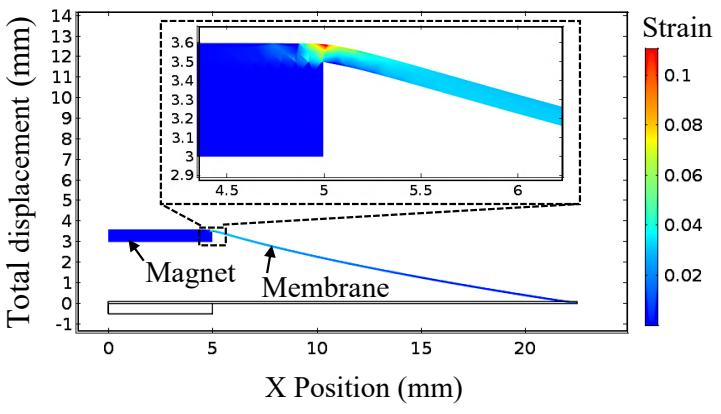

(b)

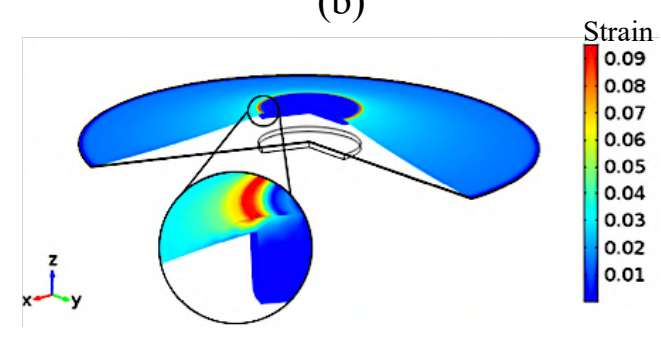

(c)

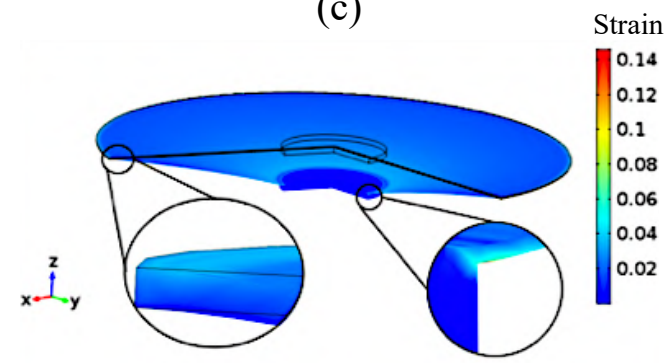

Fig. 3: Membrane deformation and strain distribution. (a) cross-section view and $3 \mathrm{D}$ view with the insets showing the strain distribution on the surface at $+3.5 \mathrm{~mm}$ (b) and $-3.5 \mathrm{~mm}$ (c) of deflection, respectively.

condition around the circular edge of the membrane was set to be fixed. The radius of the PDMS layer and the support piece were $22.5 \mathrm{~mm}$ and $5 \mathrm{~mm}$, respectively. To mimic the mechanical vibration of the experimental setup, a displacement equal to the maximum amplitude reached by the membrane in the experiment was used. This displacement of $3.5 \mathrm{~mm}$ in both directions was measured using a laser vibrometer system (Polytec OFV-5000 vibrometer controller, Polytec OFV-552 fiber vibrometer and Polytec MSA-500 microsystem analyzer). The first four eigenfrequencies of the simplified vibrating system at steady state (zero deflection) were determined. The corresponding strain distributions along the membrane with respect to the eigenfrequencies are presented in Fig. 2(b). It shows that the strain decreases with increasing the vibration frequency. Since the resulting strain amplitude varies only slightly between the different frequencies, a frequency value of $24 \mathrm{~Hz}$ was selected for the experiments, based on practical reasons, such as the stability of the liquid medium inside the container and the resistance of the membrane to damage. The strain on the upper surface of the membrane, where the hMSCs were cultured, was simulated at the maximum deflection amplitude 


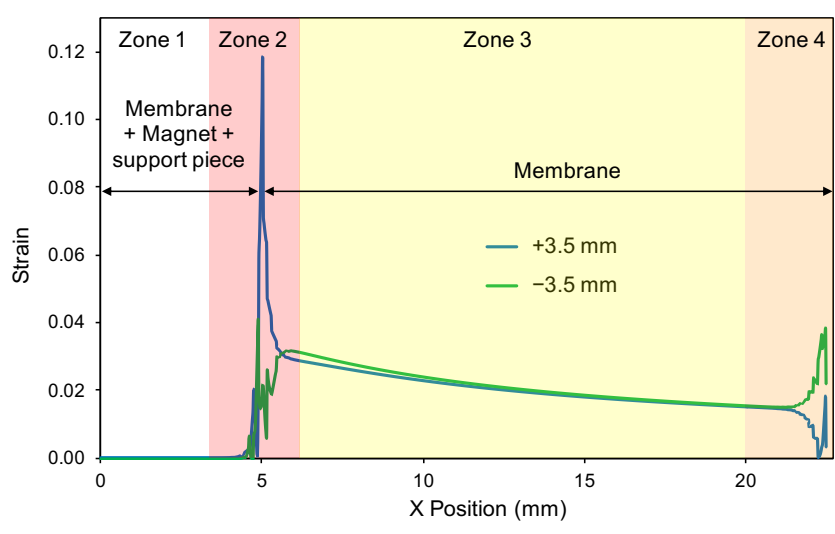

Fig. 4: Strain distribution along the membrane's upper surface when deflected in positive and negative $\mathrm{z}$-direction.

of the membrane, i.e., $\pm 3.5 \mathrm{~mm}$. Fig. 3 shows the $2 \mathrm{D}$ crosssection of the membrane deformation under displacement of $3.5 \mathrm{~mm}$ in the upper direction and the induced strain distribution along the PDMS membrane. The maximum strain is located just on top of the edge of the support piece, while the region between the support piece and the outer frame shows a gradually reducing strain amplitude towards the outer frame. A plot of the strain distribution on the membrane's upper side at $\pm 3.5 \mathrm{~mm}$ deflections with respect to the $\mathrm{x}$ position is shown in Fig. 4. From the membrane's center (at zero $\mathrm{x}$ position) to the edge of the support piece/magnet, the membrane does not undergo any strain as it is rigidly fixed to the support. Immediately after the edge of the support, the membrane is free and can undergo deformation. The highest strain of about 0.12 occurs when the magnet moves in the positive z-direction and reaches a deflection of $+3.5 \mathrm{~mm}$ at an $\mathrm{X}$-position equal to the radius of the support piece. In this zone, the membrane undergoes the largest strain. The maximum strain is considerably smaller when moving in the negative $z$-direction with a value of 0.04 , due to the existence of compressive strain in the membrane's upper surface that compensates part of the main deformation induced by the magnet. Along the major part of the membrane (zone 3), the strain decreases slightly from about 0.03 to about 0.015 in both movement directions. In the peripheral area (zone 4), the strain drops to zero, peaks at a value of 0.015 and drops to zero again, in the case of deflection in the positive z-direction. In case of deflection in the negative z-direction, it shows a peak of 0.04 before it drops back to 0.02 . The zones described above were used for analyzing the stem cell response with respect to different strain distributions. In summary, zone 1 at the center of the membrane, where the support piece is located, has zero strain. Zone 2 is the area of highest strain with an average value of $12 \%$. Zone 3 corresponds to the area of low strain with an average value of $2 \%$, located between the support piece and the edge of the membrane. Zone 4 is the area close to the edge of the membrane and is characterized by an intermediate (medium) strain of $4 \%$ on average.

The distribution of osteoblast differentiation across the membrane after 14, 17 and 21 days of incubation under constant vibration are shown in Fig. 5(a). These data indicate (a)

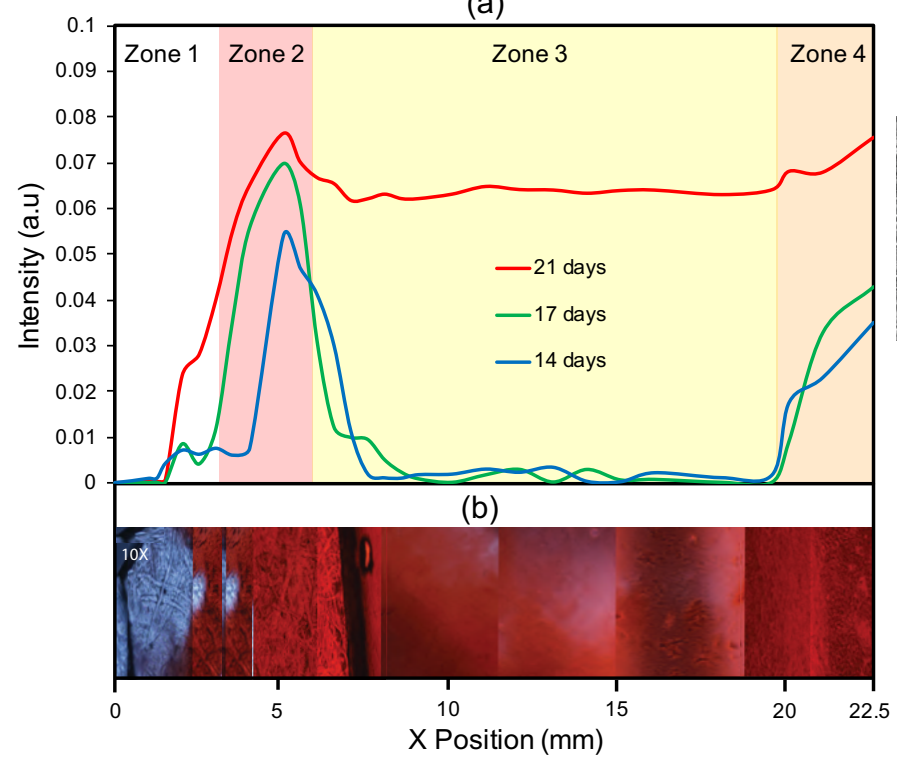

(c)

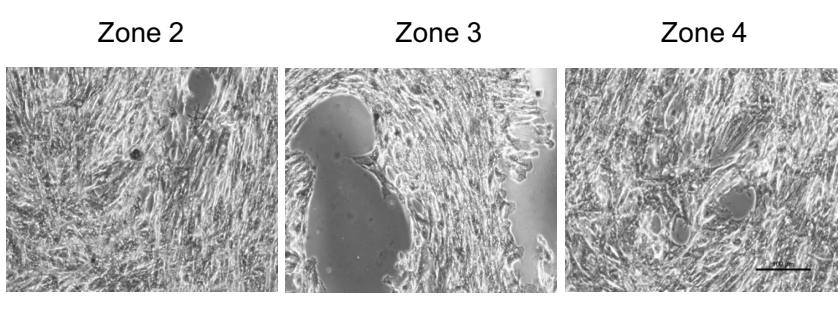

(d)

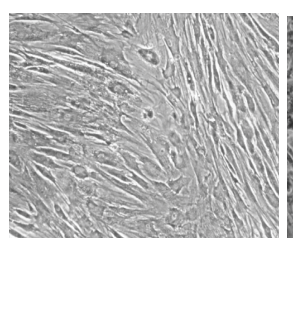

(e)

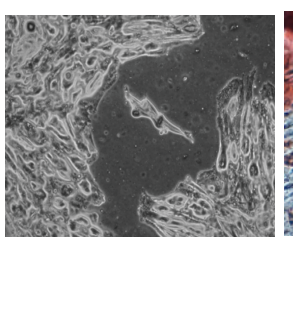

(f)

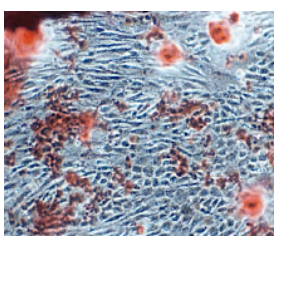

Fig. 5: (a) Alizarin S red signal measuring the distribution of hMSC differentiation into osteogenic cells across the vibrating membrane. The zones indicating the low (zone 3), medium (zone 4) and high (zone 2) strain in the membrane are indicated in the shaded colored regions. The hMSCs were incubated for 14 days (blue), 17 days (green) and 21 days (red). (b) A collage of optical microscope images of hMSCs from the center (starting at $0 \mathrm{~mm}$ ) to the outer border (ending at $22.5 \mathrm{~mm}$ ) of the membrane where hMSCs were grown for 21 days under constant vibration and subsequently stained with Alizarin $\mathrm{S}$ red. (c) Alizarin red staining of a parallel control membrane not exposed to a magnetic field vibration located in the same incubator as a control. (d) Negative control of hMSCs stained with Alizarin S red cultured on a typical tissue culture dish. (e) Negative control of hMSCs stained with Alizarin $\mathrm{S}$ red cultured on a fibronectin-coated tissue culture dish. (f) Positive control with hMSCs seeded onto a fibronectin-coated tissue culture dish and with osteogenic differentiation media (Stem Cell Technologies Inc.). All experiments were performed using hMSCs in passage 3-5. This is representative data of at least $\mathrm{n}=5$ independent experiments using $\mathrm{n}=5$ different lots of hMSCs from AllCells. 
that even as early as 14 days (blue plot) and 17 days (green plot), it is possible to detect osteogenic differentiation of hMSCs in zone 2 and zone 4 where the strain is relatively high compared to the rest of the membrane (zone 1 and zone 3). It should be noted that typical chemical induction of osteogenesis (using MesenCult Osteogenic Differentiation kit) shows little to no staining since the stimulation time was not enough to induce full differentiation. Also, no staining was observed on membranes where hMSCs were incubated without a magnetic field generated vibration (Fig. 5(c)) as well as hMSCs cultured in normal tissue culture plates (Fig. 5(d)) or tissue culture plates coated with fibronectin (Fig. 5(e)). Differentiation was also deduced using Von Kossa calcium staining and it was consistent with Alizarin red staining. Results were compared to chemical osteogenic differentiation induced using osteogenic differentiation media (Fig. 5(f)). Interestingly, immunohistochemical analysis of the membranes exposed to vibration revealed Alizarin red staining throughout zones 2,3 and 4 at 21 days indicating that there may be a positive feedback effect of those differentiated cells from zones 2 and 4 to those cells within zone 3 (Fig. 5(b)).

Overall these results suggest that osteoblast differentiation can be controlled through changes in strain imposed on the hMSCs. In addition, the effect of the strain as a stimulus appears to accelerate (by 34\%) the differentiation of hMSCs to osteoblasts, which normally needs 21 days using chemical methods (Fig. 5(f)).

\section{CONCLUSION}

The MSCs were cultured on a vibrating membrane to study their differentiation behavior as a function of strain. The membrane provides regions of different strain intensity with average values of $0,0.12,0.02$, and 0.04 . This allows the direct comparison of strain effects on the cells in each experiment. Tracking hMSCs differentiation over different incubation days indicated that the 14- and 17-days results correlate well with the membrane's strain distribution, while the results at 21 days (normal differentiation time) showed a saturation of osteoblast differentiation. The results showed that there is a positive correlation between strain and differentiation into osteogenic cells, providing a mechanism to increase the cell differentiation rate with this or a similar setup. The advantage of the proposed setup is that various parameters could be used to tailor the differentiation behavior like membrane dimensions, magnetic field amplitude or frequency.

\section{ACKNOWLEDGMENTS}

This work was funded by King Abdullah University of Science and Technology (KAUST).

\section{REFERENCES}

[1] C. L. Ross, M. Siriwardane, G. Almeida-Porada, C. D. Porada, P Brink, G. J. Christ, and B. S. Harrison, "The effect of low-frequency electromagnetic field on human bone marrow stem/progenitor cell differentiation," Stem Cell Research, vol. 15, no. 1, pp. 96-108, 2015.
[2] Y. Wang, X. Chen, W. Cao, and Y. Shi, "Plasticity of mesenchymal stem cells in immunomodulation: pathological and therapeutic implications," Nature Immunology, vol. 15, no. 11, pp. 1009-1016, 2014.

[3] E. Sykova and S. Forostyak, "Stem Cells in Regenerative Medicine," Laser Therapy, vol. 22, no. 2, pp. 87-92, 2013.

[4] G. Q. Daley and D. T. Scadden, "Prospects for Stem Cell-Based Therapy," Cell, vol. 132, no. 4, pp. 544-548, 2008.

[5] Z. Shahrokh, L. Royle, R. Saldova, J. Bones, J. L. Abrahams, et al., "Erythropoietin Produced in a Human Cell Line (Dynepo) Has Significant Differences in Glycosylation Compared with Erythropoietins Produced in CHO Cell Lines," Molecular Pharmaceutics, vol. 8, no. 1, pp. 286-296, 2010.

[6] J. I. Lopez, J. K. Mouw, and V. M. Weaver, "Biomechanical regulation of cell orientation and fate," Oncogene, vol. 27, no. 55, pp. 6981-6993, 2008.

[7] A. I. Caplan, "Adult mesenchymal stem cells for tissue engineering versus regenerative medicine," Journal of Cellular Physiology, vol. 213, no. 2, pp. 341-347, 2007.

[8] A. D. Lander, J. Kimble, H. Clevers, E. Fuchs, D. Montarras, et al., "What does the concept of the stem cell niche really mean today?," BMC Biology, vol. 10, no. 1, Sep. 2012.

[9] S. Bandyopadhyay, R. M. Austin, D. Dabbs, and C. Zhao, "Adjunctive human papillomavirus DNA testing is a useful option in some clinical settings for disease risk assessment and triage of females with ASC-H Papanicolaou test results," Archives of pathology \& laboratory medicin, vol. 132, no. 12, pp. 1874-1881, 2008.

[10] R. Schofield, "The relationship between the spleen colony-forming cell and the haemopoietic stem cell," Blood cells, 1978;4(1-2):7-25.

[11] V. Mann, C. Huber, G. Kogianni, D. Jones, and B. Noble, "The influence of mechanical stimulation on osteocyte apoptosis and bone viability in human trabecular bone," Journal of musculoskeletal \& neuronal interactions, vol. 6, no. 4, pp. 408, 2006.

[12] M. Marędziak, A. Śmieszek, K. Chrząstek, K. Basinska, and K. Marycz, "Physical Activity Increases the Total Number of BoneMarrow-Derived Mesenchymal Stem Cells, Enhances Their Osteogenic Potential, and Inhibits Their Adipogenic Properties," Stem Cells International, vol. 2015, pp. 1-11, 2015.

[13] C. Alpaslan, G. Alpaslan, and D. Uğar, "Postoperative pain control by single doses of piroxicam administered sublingually and aspirin," Journal of Marmara University Dental Faculty, vol. 2, no. 4, pp. 658664, 1997.

[14] Y. Yang, N. K. Relan, D. A. Przywara, and L. Schuger, "Embryonic mesenchymal cells share the potential for smooth muscle differentiation: myogenesis is controlled by the cell's shape," Development, vol. 126, no. 13, pp. 3027-3033, 1999.

[15] F. J. Manasek, M. Burnside, and R. E. Waterman, "Myocardial cell shape change as a mechanism of embryonic heart looping," Developmental Biology, vol. 29, no. 4, pp. 349-371, 1972.

[16] D. Ingber, "Extracellular matrix and cell shape: potential control points for inhibition of angiogenesis," Journal of cellular biochemistry, vol. 47, no. 3, pp. 236-241, 1991.

[17] S. A. Ruiz and C. S. Chen, "Emergence of Patterned Stem Cell Differentiation Within Multicellular Structures," Stem Cells, vol. 26, no. 11 , pp. 2921-2927, 2008.

[18] R. Mcbeath, D. M. Pirone, C. M. Nelson, K. Bhadriraju, and C. S Chen, "Cell Shape, Cytoskeletal Tension, and RhoA Regulate Stem Cell Lineage Commitment," Developmental Cell, vol. 6, no. 4, pp. 483-495, 2004.

[19] J. E. Perez, T. Ravasi, and J. Kosel, "Mesenchymal stem cells cultured on magnetic nanowire substrates, Nanotechnology, vol. 28, no. 5, 2016.

[20] Y. Chen, Z. Sun, Y. Li, and Y. Hong, "Osteogenic Commitment of Mesenchymal Stem Cells in Apatite Nanorod-Aligned Ceramics," ACS Applied Materials \& Interfaces, vol. 6, no. 24, pp. 21886-21893, Mar. 2014.

[21] J. Park, S. Bauer, K. V. D. Mark, and P. Schmuki, "Nanosize and Vitality: TiO2Nanotube Diameter Directs Cell Fate," Nano Letters, vol. 7, no. 6, pp. 1686-1691, 2007.

[22] S.-W. Kuo, H.-I. Lin, J. H.-C. Ho, Y.-R. V. Shih, H.-F. Chen, et al., "Regulation of the fate of human mesenchymal stem cells by mechanical and stereo-topographical cues provided by silicon nanowires," Biomaterials, vol. 33, no. 20, pp. 5013-5022, 2012.

[23] D. Liu, C. Yi, K. Wang, C.-C. Fong, Z. Wang, et al., "Reorganization of Cytoskeleton and Transient Activation of Ca2 Channels in Mesenchy- 
mal Stem Cells Cultured on Silicon Nanowire Arrays," ACS Applied Materials \& Interfaces, vol. 5, no. 24, pp. 13295-13304, 2013.

[24] D. Liu, C. Yi, C.-C. Fong, Q. Jin, Z. Wang, W.-K. Yu, D. Sun, J. Zhao, and M. Yang, "Activation of multiple signaling pathways during the differentiation of mesenchymal stem cells cultured in a silicon nanowire microenvironment," Nanomedicine: Nanotechnology, Biology and Medicine, vol. 10, no. 6, pp. 1153-1163, 2014.

[25] E. Sackmann, "How cells feel the force," Nature Physics, vol. 6, no. 6, pp. 407-408, 2010.

[26] S. Mayer-Wagner, A. Passberger, B. Sievers, J. Aigner, B. Summer, et al., "Effects of low frequency electromagnetic fields on the chondrogenic differentiation of human mesenchymal stem cells," Bioelectromagnetics, vol. 32, no. 4, pp. 283-290, 2010.

[27] J. H. C. Wang and B. P. Thampatty, "Chapter 7 Mechanobiology of Adult and Stem Cells," International Review of Cell and Molecular Biology, pp. 301-346, 2008.

[28] G. Osol, "Mechanotransduction by Vascular Smooth Muscle," Journal of Vascular Research, vol. 32, no. 5, pp. 275-292, 1995.

[29] P. F. Davies, "Flow-mediated endothelial mechanotransduction," Physiological Reviews, vol. 75, no. 3, pp. 519-560, Jan. 1995.

[30] S. Chien, "Mechanotransduction and endothelial cell homeostasis: the wisdom of the cell," American Journal of Physiology-Heart and Circulatory Physiology, vol. 292, no. 3, 2007.

[31] Y. K. Luu, E. Capilla, C. J. Rosen, V. Gilsanz, J. E. Pessin, S. Judex, and C. T. Rubin, "Mechanical Stimulation of Mesenchymal Stem Cell Proliferation and Differentiation Promotes Osteogenesis While Preventing Dietary-Induced Obesity," Journal of Bone and Mineral Research, vol. 24, no. 1, pp. 50-61, 2009.

[32] C. Zhang, Y. Lu, L. Zhang, Y. Liu, Y. Zhou, Y. Chen, and H. Yu, "Influence of different intensities of vibration on proliferation and differentiation of human periodontal ligament stem cells," Archives of Medical Science, vol. 3, pp. 638-646, 2015.

[33] E. Lau, W. D. Lee, J. Li, A. Xiao, J. E. Davies, Q. Wu, L. Wang, and L. You, "Effect of low-magnitude, high-frequency vibration on osteogenic differentiation of rat mesenchymal stromal cells," Journal of Orthopaedic Research, vol. 29, no. 7, pp. 1075-1080, 2011.

[34] E. Saino, L. Fassina, S. V. Vlierberghe, M. Avanzini, P. Dubruel, G. Magenes, L. Visai, and F. Benazzo, "Effects of Electromagnetic Stimulation on Osteogenic Differentiation of Human Mesenchymal Stromal Cells Seeded onto Gelatin Cryogel," International Journal of Immunopathology and Pharmacology, vol. 24, pp. 1-6, 2011.

[35] F. Luo, T. Hou, Z. Zhang, Z. Xie, X. Wu, and J. Xu, "Effects of Pulsed Electromagnetic Field Frequencies on the Osteogenic Differentiation of Human Mesenchymal Stem Cells," Orthopedics, vol. 35, no. 4, Jan. 2012.

[36] K. Marycz, D. Lewandowski, K. A. Tomaszewski, B. M. Henry, E. B. Golec, and M. Marędziak, "Low-frequency, low-magnitude vibrations (LFLM) enhances chondrogenic differentiation potential of human adipose derived mesenchymal stromal stem cells (hASCs)," PeerJ, vol. 4, 2016.

[37] J. H. Edwards and G. C. Reilly, "Vibration stimuli and the differentiation of musculoskeletal progenitor cells: Review of results in vitro and in vivo," World Journal of Stem Cells, vol. 7, no. 3, pp. 568-582, 2015.

[38] H.-Y. Lin and Y.-J. Lin, "In vitro effects of low frequency electromagnetic fields on osteoblast proliferation and maturation in an inflammatory environment," Bioelectromagnetics, vol. 32, no. 7, pp. 552-560, 2011.

[39] J. Shi, D. Ahmed, X. Mao, S.-C. S. Lin, A. Lawit, and T. J. Huang, "Acoustic tweezers: patterning cells and microparticles using standing surface acoustic waves (SSAW)," Lab on a Chip, vol. 9, no. 20, p. 2890, 2009.

[40] A. Neild, S. Oberti, G. Radziwill, and J. Dual, "Simultaneous positioning of cells into two-dimensional arrays using ultrasound," Biotechnology and Bioengineering, vol. 97, no. 5, pp. 1335-1339, 2007.

[41] M.-T. Tsai, W.-J. Li, R. S. Tuan, and W. H. Chang, "Modulation of osteogenesis in human mesenchymal stem cells by specific pulsed electromagnetic field stimulation," Journal of Orthopaedic Research, vol. 27, no. 9, pp. 1169-1174, 2009.

[42] M.-C. Bélanger and Y. Marois, "Hemocompatibility, biocompatibility, inflammatory andin vivostudies of primary reference materials lowdensity polyethylene and polydimethylsiloxane: A review," Journal of Biomedical Materials Research, vol. 58, no. 5, pp. 467-477, 2001.
[43] O. D. Roure, A. Saez, A. Buguin, R. H. Austin, P. Chavrier, et al., "Force mapping in epithelial cell migration," Proceedings of the National Academy of Sciences, vol. 102, no. 7, pp. 2390-2395, 2005. 